\title{
Ultrasonic Radial Simulation Research of Solid Particle Distribution of Segregation Flow in Gas-solid Two Phase Flow
}

\author{
Yueming Wang ${ }^{1}$, Guodong $\mathrm{Yao}^{2}$, Yaoyao Zhang ${ }^{1}$, Meng Liu ${ }^{1}$ and Pengcheng $\mathrm{Ge}^{1}$ \\ ${ }^{1}$ School of information engineering, Inner Mongolia University of Science and Technology, Baotou, Inner Mongolia, 014010 , \\ China \\ ${ }^{2}$ Tech. PMT, CNCEC Sixth Construction Company, Xiangyang, Hu Bei, 441000, China
}

\begin{abstract}
As for its application and universality, the research of gas-solid two-phase flow becomes popular, and an ultrasonic sensor is used to test gas- solid two -phase flow. By COMSOL software, a radial simulation model is set up to study particle distribution in radial section and their sensitivity. And also, the signals received by the ultrasonic sensor in different frequency and locations are analyzed. Thus, it is helpful to measure gas-solid two-phase flow in the ultrasonic method.
\end{abstract}

Keywords-gas solid two phase flow; ultrasonic sensor; particle distribution; segregation flow; simulation

\section{INTRODUCTION.}

Ultrasonic measurement aims to detect the transmission velocity in gas-solid two phase flow and particle density $[1,2]$. Because Ultrasonic is of mechanical waves and its transmission velocity is determined largely by medium, ultrasonic measurement is used to measure coal powder density as to different ultrasonic transmission velocity in different solid density [3]. Due to the fact that ultrasonic measurement is non-contacted and can be used to measure the transient velocity distributions, it has found some application in fluid in recent years and some achievements have been made. At abroad, it is used to test gas fluid and some ultrasonic measurement devices are mature in the Netherlands, Germany, the United States, South Korea and so on. At home, ultrasonic liquid meters are more mature than gas ones, and little research is done in gas ones except in some universities and institutions[4,5], let alone that in gas-solid two phase flow velocity. Mingxu $\mathrm{Su}$ et al have done some research about ultrasonic attenuation and velocity of ultrafine particles suspension liquid. [6,7] Some scholars in Tianjin University have done some full research in ultrasonic measurement to gas-solid two phase flow [8-10].

In this paper, with COMSOL software, a simulation model is built to measure potential distribution and current density as well as sensitivity in the gas-solid two phase flow. The simulation result supports the theory of measuring gas-solid two-phase flow in the ultrasonic method.

\section{The Simulation Ultrasonic Model Containing SOLID PHASE PARTICLES IN PIPE}

The ultrasonic transmission is studied as followed when solid phase particles are conveyed in the pneumatic conveying pipe. When gas-solid two phase flow passes through a pipe bend or in some special cases, gas-solid two phase flow is segregated. Solid phase particles are distributed regularly in different radial sections to study ultrasonic sensitivity in different segregations and frequency.

\section{A. Introduction of Simulation Modeling}

Based on ultrasonic measurement of solid phase particles in pipe, a simulation model of gas-solid two phase flow is shown in Figure 1. In Figure1 (a), the pipe radius is set to $\mathrm{R}(\mathrm{R}=150 \mathrm{~mm})$, and the section diameter of ultrasonic sensors of emission and reception is set to $10 \mathrm{~mm}$. Usually, sensors are composited by flexible compound piezoelectric materials $\mathrm{T}$ and $\mathrm{H}$, with $\mathrm{T}$ of the emitting terminal, $\mathrm{H}$ of the receiving terminal, and their thickness of $4.5 \mathrm{~mm}$. The solid phase particles in pipe are set to the diameter of $d 1=2 \mathrm{~mm}$. T and $\mathrm{H}$ are located on $\mathrm{Y}$ axis, and $\mathrm{X}$ axis and $\mathrm{Y}$ axis are composited into a rectangular coordinate system. The gap length is $30 \mathrm{~mm}$ between the centers of particles which are distributed in linear array, shown in Figure1 (b, c). In Figure1 (d), with ultrasonic frequency of $30 \mathrm{kHz}$ and the mesh generation, a simulated mesh dissection model is achieved in the form of triangular mesh. Under the rule of the maximum mesh size $\leq \lambda / \mathrm{N}$, (where $\mathrm{N}=10$ and the maximum mesh size $\leq 1 / 10$ of the ultrasonic wavelength), fine geometric properties and wavelength are parsed. In the simulation experiment, solid phase particles are distributed accordingly (decreasing or increasing particles) to simulate various segregations of gas-solid two phase flow in pipe practically.

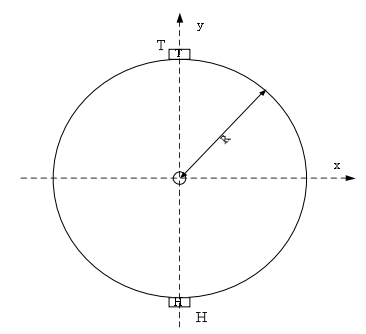

(a)

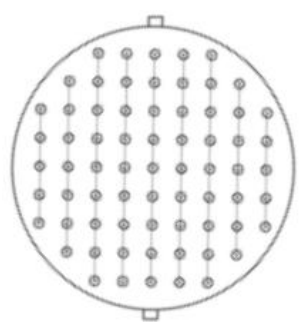

(b) 


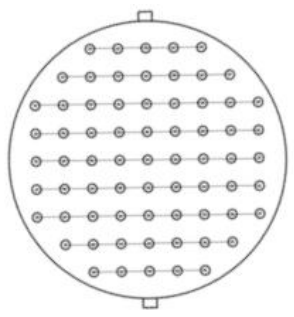

(c)

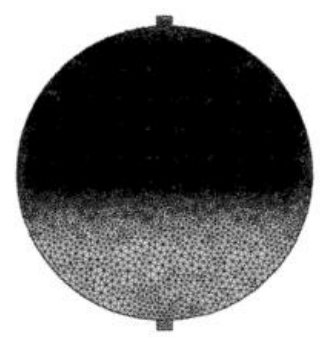

(d)
FIGURE I. COMSOL SIMULATION MODEL

B The Coupling Equation of Electricity - Structure - Acoustic System.

In the simulation experiment, the sound propagation equation is constructed by the momentum conservation equation and energy conservation equation of the Navier-Stokes equation. Supposing particle sizes are kept constant and lossless, the pipe is adiabatic, viscous effects are neglected, linear isentropic state equations are used, and sound field is depicted by a variable of sound pressure, thus Equation (1) is obtained by solving the wave equation.

$$
\frac{1}{\rho c^{2}} \frac{\partial^{2} p}{\partial t^{2}}+\nabla \cdot\left(-\frac{1}{\rho}(\nabla p t-q)\right)=Q
$$

where $\rho$ stands for the density of material, t represents time, $\mathrm{p}$ is the sound pressure, and $\nabla$ is Laplace operator. Q and $Q$ are acoustic dipole source and single pole source respectively, and signals can be converted into a series of harmonic components by Fourier transform. A frequency can be solved once in frequency domain by wave equation. General harmonic component is as Equation (2):

$$
\mathrm{p}(\mathrm{x}, \mathrm{t})=\mathrm{p}(\mathrm{x}) \sin (\omega \mathrm{t})
$$

where harmonic component peak is $\mathrm{p}(\mathrm{x})$, more generally it is Equation (3):

$$
\mathrm{p}(\mathrm{x}, \mathrm{t})=\mathrm{p}(\mathrm{x})
$$

Physical value of actual sound pressure is the real part in Equation (3). With sound pressure supposed above, transient wave equation shapes in Helmholtz Equation (4):

$$
\nabla \cdot\left(-\frac{1}{\rho}(\nabla p t-q)\right)-\frac{w}{\rho c^{2}} p=Q
$$

In the model, flow media is distributed evenly, and source terms of q and Q equal zero, thus Helmholtz Equation (4) results in a simple solution of plane wave as Equation (5):

$$
p=p_{0} e^{i(\omega t-k \cdot x)}
$$

Where $\mathrm{p}$ stands for amplitude and wave goes with the angular frequency of $\mathrm{W} \omega$ and $\mathrm{K}=|\mathrm{k}|$ in the direction of vector.Structural mechanics equation is expressed as Equation (6):

$$
\rho \frac{\partial^{2} u}{\partial t^{2}}=\nabla \cdot s+F
$$

Where $\rho$ stands for piezoelectric material density, $\mathrm{u}$ is displacement, Fv is body force, and $\mathrm{s}$ is stress. Maxwell's equation of the Electric on piezoelectric material is expressed as Equation (7):

$$
\nabla \cdot D=p v
$$

where $\mathrm{D}$ is the electric displacement, and $\rho_{V}$ is the volume charge density.

In the simulation experiment, the compound piezoelectric material $\mathrm{T}$ loaded with an instantaneous sine excitation voltage of $223.72 \mathrm{~V}$ is shown in Figure 2(a), and the time domain signals of excitation voltage are shown in Figure 2(b)

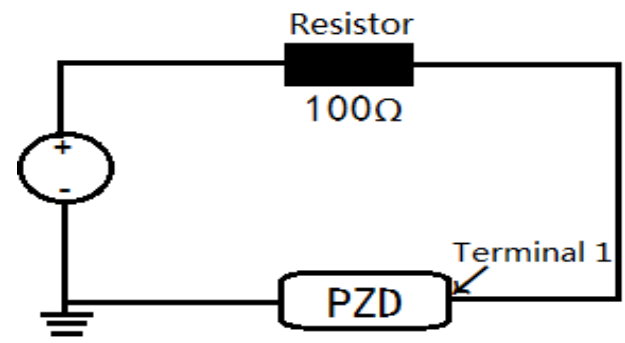

(a)

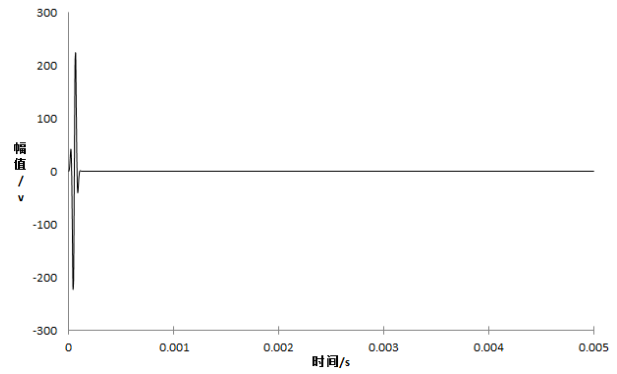

(b)

FIGURE II. EQUIV ALENT CIRCUIT DIAGRAM AND SIMULATED DIAGRAM OF EXCITATION VOLTAGE SIGNALS

\section{The Simulation PRocess AND ANALYSIS}

In the simulation experiment of gas-solid two phase flow, particles set with the diameter of $2 \mathrm{~mm}$ and the particle gap of $30 \mathrm{~mm}$ are lined in the pipe, and the lines are increased one by one, with frequency of $20 \mathrm{kHz}, 30 \mathrm{kHz}, 100 \mathrm{kHz}$. In the experiment, ultrasonic transmission direction is set as $\mathrm{Y}$ axis, and the vertical direction of ultrasonic transmission is set as $\mathrm{X}$ axis. The particle lines are increased one by one along $X$ axis 
and $\mathrm{Y}$ axis separately to form different radical sections. Two COMSOL diagrams of sound pressure at $6 \mathrm{e}-4 \mathrm{~s}$ are shown in Figure $3(\mathrm{a}, \mathrm{b})$, with 5 particle lines parallel or vertical to ultrasonic transmission direction, and frequency of $30 \mathrm{kHz}$.

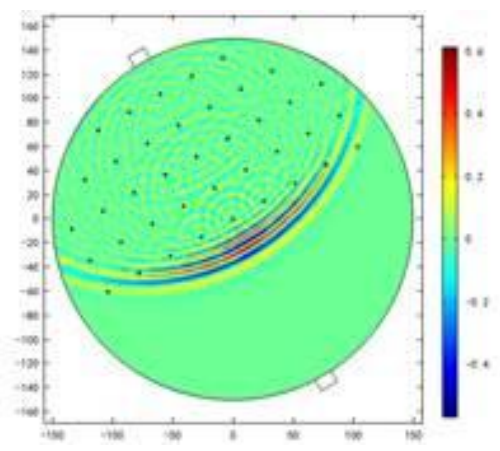

(a)

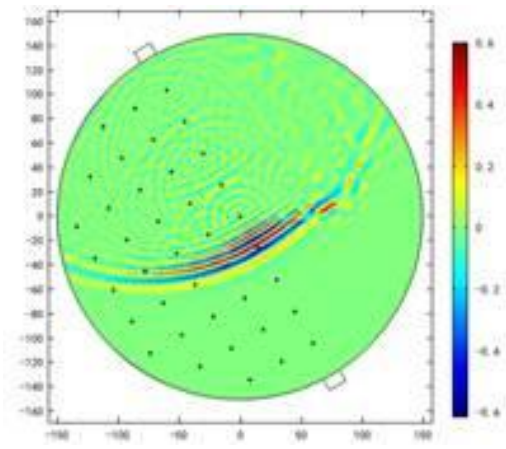

(b)

FIGURE III. THE SIMULATION OF SOUND PRESSURE DISTRIBUTION IN RADIAL SECTION IN THE PIPE

Seen from the simulation diagram above, gas-flow two phase flow does affect ultrasonic transmission, namely with sound pressure amplitude (data) varying near particles. Further analysis is done to signal strength.

In the simulation experiment, different sound pressure data are obtained with different particle locations (particle lines increased from 1 to 9), under the different frequencies of $20 \mathrm{kHz}, 30 \mathrm{kHz}, 100 \mathrm{kHz}$ separately. Sound pressure diagrams with different particle locations are shown in Figure 4.

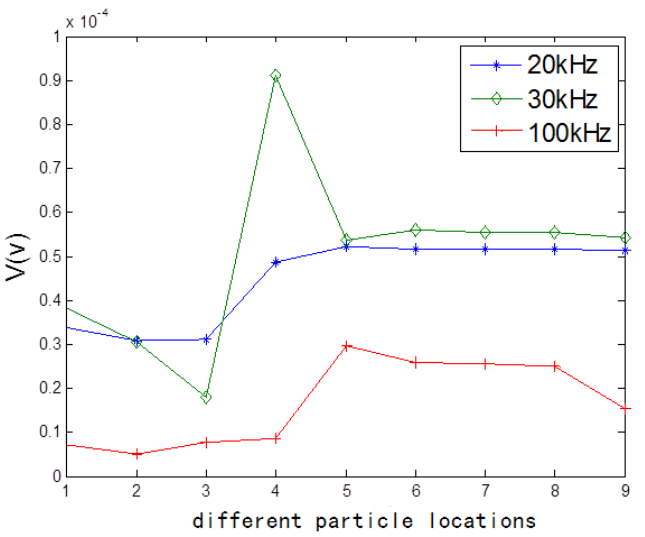

(a)

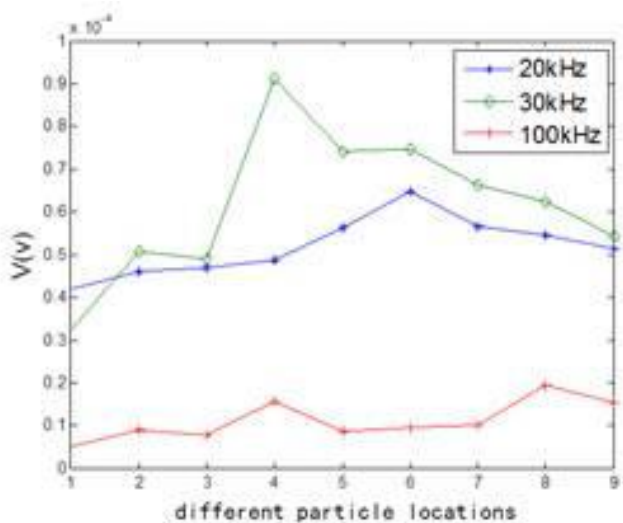

(b)

FIGURE IV. SOUND PRESSURE DIAGRAMS WITH DIFFERENT PARTICLE LOCATIONS

In Figure 4(a), Y axis stands for sound pressures while $\mathrm{X}$ axis is particle lines parallel to ultrasonic transmission direction, and in Figure 4(b), Y axis stands for sound pressures while $\mathrm{X}$ axis is particle lines vertical to ultrasonic transmission direction. In Figure $4(a, b)$, three curves are sound pressures with different particle locations under the different frequencies of $20 \mathrm{kHz}, 30 \mathrm{kHz}, 100 \mathrm{kHz}$ separately. So, based on Figure 4(a, b), a conclusion is achieved that less particle lines are in gas-solid two phase flow in pipe, weaker ultrasonic signals are, especially with particle lines vertical to ultrasonic transmission direction. Furthermore, when particle lines are increased beyond half of the pipe, ultrasonic signal strength is near to be constant.

\section{CONCLUSION}

In the paper, by COMSOL software, a radial simulation model of ultrasonic transmission in the gas-solid two-phase flow in pipe is set up. From the simulation, voltage signals which the receiver gets with different particle distribution and different frequencies are analyzed, contributing to measure gas-solid two-phase flow in the ultrasonic method.

\section{ACKNOWLEDGMENT}

This work is partially supported by the National Natural Science Foundation of China(61463042),Natural Science Foundation of Inner Mongolia (2016MS0611), Inner Mongolia university of science \&technology outstanding youth Foundation(NO.2016YQL09) and Inner Mongolia university of science \&technology Innovation Foundation(2016QDL-S16)

\section{REFERENCES}

[1] TIAN Chang,SU Ming-Xu, CAI Xiao-Shu.Particle concentration in gas-solid two-phase flow by ultrasonic methods[J],2013, 34(8): 1487-1490.

[2] Sun Jian-guo, Jin ying-zi, Jin Xi-dong. The supersonic gas-solid two-phase flow simulation of ratio values under different pressure. Journal of Zhejiang Sci-Tech University, 2013,30 (3): 354-357.

[3] F R M Yunus, R A Rahim. Simulation study ofelectrode size in air-bubble detection for dual-mode integrated electrical resistance and ultrasonic transmission tomography. Powder Technology,2014,238 (2) :345-356. 
[4] LI Qing,JIA Jing-pu. Design and Study of Ultrasonic Flowmeterfor High-temperature Flow Measure-ment[J]. Coal Technology, 2012.31 (1):209-211.

[5] BAO Li-sha,LIANG Jun-ting,LU Jie.Mathematic model of multi-path ultrasonic gas flowmeter[J].T-echnical Acoustics,2010,29(4),393-395.

[6] SU Ming-Xu, CAI Xiao-Shu.The numerical study of acoustical attenuation and velocity in the sus-pension of superfine particles[J].ACTA ACUSTICA,2002;27(3):218-222.

[7] Hou Huaishu,Su Mingxu,Cai Xiaoshu.Study on measurement of ultrasonic attenuation and velocity spectra in superfine particle suspensions[J].Chinese Journal of Scientific Instrument,2010;241- 246.

[8] Dandan Zheng, Huirang Hou, Tao Zhang, Research and realization of ultrasonic gas flow rate measurement based on ultrasonic exponential model, Ultrasonics, 2016, 67: 112-119 (SCI)

[9] Dandan Zheng, Dan Zhao, Jianqiang Mei, Improved numerical integration method for flowrate of ultrasonic flowmeter based on Gauss quadrature for non-ideal flow fields, Flow Measurement and Instrumentation, 2015, 41(3): 28-35 (SCI)

[10] Huirang Hou, Dandan Zheng, Laixiao Nie, Gas ultrasonic flow rate measurement through genetic-ant colony optimization based on ultrasonic pulse received signal model, Measurement Science and Technology, 2015, 26(4): 045005 (SCI) 